\title{
Energy Conversion during Microwave Sintering of a Multiphase Ceramic Surrounded by a Susceptor
}

\author{
Jacob Lasri, ${ }^{\dagger}$ Peelamedu D. Ramesh, ${ }^{\dagger} \S$ and Levi Schächter ${ }^{\dagger}$ \\ Departments of Electrical Engineering and Materials Engineering, Technion-Israel Institute of Technology, Haifa 32000, Israel
}

\begin{abstract}
A quasi-analytic model has been developed to examine energy conversion during the microwave sintering of a ceramic that is surrounded by a susceptor. Low-loss ceramics, such as $\mathrm{ZrO}_{2}$, couple poorly with microwave radiation at low temperatures; however, because the dielectric loss usually increases rapidly as temperature increases, coupling improves dramatically at high temperatures. To improve heat transfer at low temperatures, susceptors are used. Three processes of energy flow are considered: microwave absorption due to dielectric losses, blackbody radiation, and heat convection. As expected, the susceptor $(\mathrm{SiC})$ heats rapidly, relative to the ceramic $\left(\mathrm{ZrO}_{2}\right)$, at low temperatures; however, the ceramic attains higher temperatures after a prolonged period of microwave exposure. Below a critical temperature $\left(800^{\circ} \mathrm{C}\right)$, the primary heattransfer mechanism to the $\mathrm{ZrO}_{2}$ is blackbody radiation from the susceptor. Above this temperature, microwave radiation is the main source that contributes to the temperature increase of the ceramic. The results of the simulation are in reasonable agreement with recent experimental data.
\end{abstract}

\section{Introduction}

$\mathrm{C}$ ERAMIC sintering is a fast-growing area of microwave applications. ${ }^{1}$ The advantages over conventional heating include rapid heating rates, uniformity, and low power requirements. Zirconia $\left(\mathrm{ZrO}_{2}\right)^{2}$ and alumina $\left(\mathrm{Al}_{2} \mathrm{O}_{3}\right)^{3}$ are low-loss ceramics that couple poorly to microwave radiation at low temperatures. However, because dielectric loss usually increases as temperature increases (by five orders of magnitude in the case of $\mathrm{ZrO}_{2}$ when the temperature increases from $300^{\circ} \mathrm{C}$ to $1500^{\circ} \mathrm{C}$ ), ceramics can be made absorptive by increasing their temperature. A simple technique to implement such heating is to surround the specimen with a susceptor; silicon carbide $(\mathrm{SiC})^{4}$ is commonly used for this purpose. Several configurations have been suggested for heating $\mathrm{ZrO}_{2}$ and $\mathrm{Al}_{2} \mathrm{O}_{3}$ with a SiC susceptor. Dé et al..$^{5}$ used a SiC-lined susceptor that surrounded the specimen to sinter $\mathrm{Al}_{2} \mathrm{O}_{3}$ compacts. Janney et al. ${ }^{6}$ suggested an array of $\mathrm{SiC}$ rods to sinter $\mathrm{ZrO}_{2},{ }^{6}$ and Ramesh et al. ${ }^{7}$ used a hybrid microwave-heating configuration for $\mathrm{ZrO}_{2}$ and $\mathrm{Al}_{2} \mathrm{O}_{3}$ compacts.

In this study, a simple model has been used to investigate energy flow during the sintering of a small $\mathrm{ZrO}_{2}$ sample that was surrounded by a susceptor. Contrary to other models ${ }^{8}$ where multiple reflections are ignored, these reflections have been considered in the present study because they have an important

J. J. Petrovic-contributing editor

\footnotetext{
Manuscript No. 189624. Received January 15, 1999; approved June 10, 1999.

Supported by the Israel Ministry of Science and the Fund for Promotion of Research at the Technion.

${ }^{\top}$ Dept. of Electrical Engineering.

Dept. of Materials Engineering.

${ }^{\S}$ Now at Institut für Keramik im Maschinebau, Universitat Karlsruhe, D-76131 Karlsruhe, Germany.
}

role, because of (i) spatial discontinuities of the dielectric coefficient in various regions and (ii) rapid variations of the dielectric coefficient, as a function of temperature. Figure 1 shows a schematic illustration of the system. $\mathrm{ZrO}_{2}$ is the ceramic to be sintered and is located in the center of the system; $\mathrm{SiC}$ acts as a susceptor, whereas $\mathrm{Al}_{2} \mathrm{O}_{3}$ acts as an insulator that confines the heat to the vicinity of the $\mathrm{ZrO}_{2}$. Note that the analysis that follows is suitable for any low-loss ceramic when the dependence of the complex dielectric coefficient on temperature is known. For $\mathrm{ZrO}_{2}$, $\mathrm{SiC}$, and $\mathrm{Al}_{2} \mathrm{O}_{3}$, the dependence is shown in Fig. 2; this parametric dependence $(\varepsilon(T))$ tacitly will be assumed to be known, regardless of the physical or chemical mechanism that controls the specific behavior. The temperature dependence of the dielectric coefficient clearly has a major role in the energy-conversion process, either directly or indirectly via reflections. Our goal in this study is to determine the temperature dynamics (spatial and temporal variations) for all regions in space and examine the effect of geometric parameters; all the other variables can be established after the temperature is known.

Microwave radiation is the only source of external power; to evaluate the temperature dynamics, it is averaged over time because any temperature-time variations are much slower than the typical variation of the radiation field. Energy flow between the various regions is assumed to be controlled by two nonlinear processes: blackbody radiation and heat convection. To simulate such a system, a theoretical model has been formulated. Simulations indicate that the energy transfer may be divided into two domains: (i) below a critical temperature $T_{\mathrm{c}}$, the temperature of the $\mathrm{ZrO}_{2}$ and $\mathrm{Al}_{2} \mathrm{O}_{3}$ varies slowly, whereas that of the $\mathrm{SiC}$ increases rapidly; (ii) at temperatures above $T_{\mathrm{c}}$, the temperature of the $\mathrm{ZrO}_{2}$ steeply increases, overtaking the temperature of the SiC. $T_{\mathrm{c}}$ is defined by the temperature of the ceramic being equal to that of the susceptor and occurs at a time $\tau_{\mathrm{c}}$. For the same time, the $\mathrm{Al}_{2} \mathrm{O}_{3}$ insulator shows only a small increase in temperature. The values of $T_{\mathrm{c}}$ and $\tau_{\mathrm{c}}$ are dependent on the volume of the $\mathrm{ZrO}_{2}$ sample and its emissivity (the latter of which is defined as the ratio of the total emissive power of a material to that of a blackbody at the same temperature). This quasi-analytic approach makes it possible to determine the relative influence of the blackbody radiation and

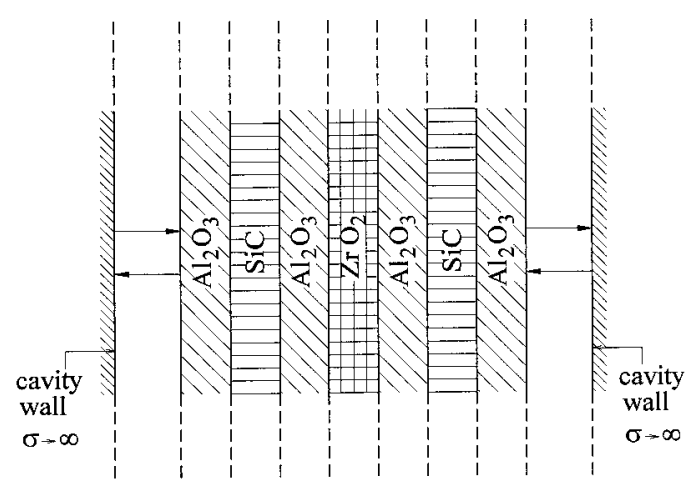

Fig. 1. Schematic depiction of the cavity configuration for sintering $\mathrm{ZrO}_{2}$. 

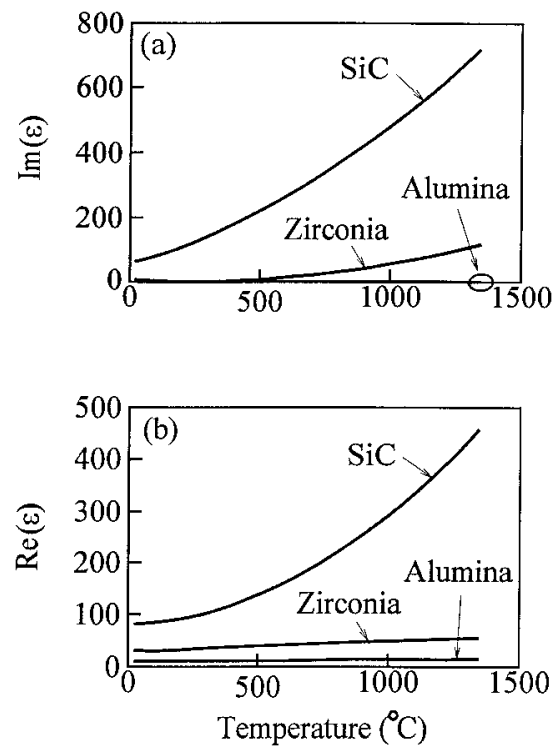

Fig. 2. Plots of the dielectric coefficient of the materials ((a) imaginary part (losses) and (b) real part) versus temperature.

convection at any instant. In particular, the blackbody radiation is shown to be the mechanism that is responsible for the dramatic increase in heat loss of the $\mathrm{ZrO}_{2}$ at high temperatures. Multiple microwave reflections between the various regions have an important role, because the dielectric coefficient of each layer varies dramatically with temperature.

\section{Theoretical Model}

Ceramics usually are sintered in multimode cavities, and the sample size usually is less than one vacuum wavelength. Exact solution of the three-dimensional (3-D) electromagnetic and thermal problem requires the application of various assumptions to simplify the highly nonlinear problem. Our goal in this study is to investigate the main features of the power flow, assuming two heat-transfer processes: blackbody radiation and convection. In addition, we consider the multiple-reflections process that is associated with layers that have different dielectric coefficients. Each layer in Fig. 1 is considered to be very thin (relative to the wavelength); thus, spatial variations are negligible, as is the contribution of heat conduction to power transfer. In the evaluation of the electromagnetic field, we consider the multiple reflections that are associated with dielectric discontinuities and temperature differences between various layers. This consideration is important in cases when simple exponential decay ${ }^{8}$ of the amplitude is not an accurate description of the propagation of the electromagnetic wave from the cavity into the various dielectric regions. The present one-dimensional (1-D) model corresponds to the cross section of an actual system: ${ }^{7}$ the sample to be sintered $\left(\mathrm{ZrO}_{2}\right)$ is located at the center of the system, separated from the susceptor by $\mathrm{Al}_{2} \mathrm{O}_{3}$, and the susceptor, in turn, is isolated from the surroundings by an additional layer of $\mathrm{Al}_{2} \mathrm{O}_{3}$.

The conversion of microwave energy to thermal energy is controlled by the relation

$$
C_{\rho} \rho\left(\frac{\partial T}{\partial t}\right)=\nabla(\kappa \nabla T)+\dot{Q}
$$

where $C_{\rho}$ is the heat capacity per unit volume, $\rho$ the mass density, $T$ the temperature, $t$ the time, $\kappa$ the Boltzmann constant, and $\dot{Q}$ the instantaneous rate of heat transfer. For simplicity, we have assumed a uniform temperature in each of the material layers, tacitly assuming a thin sample. Even if, in practice, the layer is thick, for simulation purposes, it can be divided into a set of thin layers and the temperature gradient across each layer can be neglected. In the specific case that has been studied here, the samples are relatively small, in comparison to the microwave wavelength; this assumption is justified by the good agreement with experimental results, as will be described in section III. After Eq. (1) is integrated, we have

$$
C_{\mathrm{\rho}} \rho V\left(\frac{\mathrm{d} T}{\mathrm{~d} t}\right)=P_{\mathrm{T}}
$$

where $P_{\mathrm{T}}$ represents the total power flowing in or out of the volume $V$. Three separate power sources contribute to $P_{\mathrm{T}}$. The first is the time-averaged microwave power that is absorbed by a given volume $\left(\left\langle P_{\mathrm{mw}}\right\rangle\right)$ :

$$
\begin{aligned}
\left\langle P_{\mathrm{mw}}\right\rangle=\frac{1}{2} \omega \varepsilon^{\prime \prime}\left[|A|^{2} \exp (-q d) \frac{\sinh (q d)}{q}\right. & \\
+|B|^{2} \exp (q d) \frac{\sinh (q d)}{q} & +A B^{*} \frac{1-\exp (-2 j k d)}{2 j k} \\
& \left.+A^{*} B \frac{\exp (2 j k d)-1}{2 j k}\right]
\end{aligned}
$$

where $\omega$ is the angular frequency at an operating frequency of $f=$ $2.45 \mathrm{GHz}(\omega=2 \pi f), \varepsilon^{\prime \prime}$ the imaginary part of the dielectric coefficient, and $d$ the material width; $k$ and $q$ are the real and the imaginary part of the term $(\omega / c) \varepsilon^{1 / 2}$, respectively (where $c$ is the light velocity), and $j$ is the square root of -1 . The $A B$ matrix term represents the amplitudes of the two waves bouncing between the two edges of each volume, where $A$ is the forward wave amplitude and $B$ is the backward wave amplitude, so we can assign each layer an index $\nu$. The electric field in each layer is assumed to be given by

$$
E_{\nu}=A_{\nu} \exp \left[-j\left(\frac{\omega}{c}\right) \varepsilon_{\nu}^{1 / 2} x\right]+B_{\nu} \exp \left[j\left(\frac{\omega}{c}\right) \varepsilon_{\nu}^{1 / 2} x\right]
$$

At any instant, the amplitudes are calculated assuming that the dielectric coefficient of each layer is known, as well as the quality factor and the power injected into the cavity by the microwave generator. This assumption is fully justified, because temperature variations occur within minutes, whereas the radio-frequency (rf) field varies on a subnanosecond level. Practically, the amplitudes are expressed in terms of transfer matrices of the form

$$
\begin{aligned}
& \left(\begin{array}{l}
A \\
B
\end{array}\right)_{\nu}=\left(\begin{array}{cc}
\exp \left[-j\left(\frac{\omega}{c}\right) \varepsilon_{\nu}^{1 / 2} d_{\nu}\right] & 0 \\
0 & \exp \left[j\left(\frac{\omega}{c}\right) \varepsilon_{\nu}^{1 / 2} d_{\nu}\right]
\end{array}\right) \\
& \cdot\left(\begin{array}{cc}
\frac{1}{2}\left[1+\left(\frac{\varepsilon_{\nu-1}}{\varepsilon_{\nu}}\right)^{1 / 2}\right] & \frac{1}{2}\left[1-\left(\frac{\varepsilon_{\nu-1}}{\varepsilon_{\nu}}\right)^{1 / 2}\right] \\
\frac{1}{2}\left[1-\left(\frac{\varepsilon_{\nu-1}}{\varepsilon_{\nu}}\right)^{1 / 2}\right] & \frac{1}{2}\left[1+\left(\frac{\varepsilon_{\nu-1}}{\varepsilon_{\nu}}\right)^{1 / 2}\right]
\end{array}\right)\left(\begin{array}{l}
A \\
B
\end{array}\right)_{\nu-1}
\end{aligned}
$$

The second power-transfer contribution is the time-averaged power that results from blackbody radiation emitted at a given temperature. This contribution, $\left\langle P_{\text {rad }}^{(\nu)}\right\rangle$, is defined as

$$
\left\langle P_{\mathrm{rad}}^{(\nu)}\right\rangle=S_{i} \sum_{i \neq \nu} \sigma T_{i}^{4} \alpha_{i \rightarrow \nu}-S_{\nu} \sigma T_{\nu}^{4}
$$

where the indexes $i=1,2, \ldots$ and $v=1,2, \ldots$ represent the region in space, $S$ represents the material area, and $\sigma$ is the Stefan-Boltzmann coefficient $\left(\sigma=c \pi k_{\mathrm{B}}^{4} /\left[15(h c)^{3}\right]\right)$. The term $\alpha_{i \rightarrow \nu}$ is an absorption factor $\left(\alpha_{i \rightarrow \nu} \leq 1\right)$ that represents the actual average power that is absorbed by material layer $v:^{9}$

$$
\alpha_{i \rightarrow \nu}=\frac{1}{\frac{1}{\xi_{i \rightarrow \nu}}+\frac{1}{\hat{\varepsilon}_{i}}+\frac{1}{\hat{\varepsilon}_{\nu}}-2}
$$

where $\hat{\varepsilon}$ represents the material emissivity and $\xi_{i \rightarrow v}$ is a factor that represents the actual power incident on layer $v\left(\xi_{i \rightarrow v} \leq 1\right)$. 
The third contribution to power transfer is the power-flow balance that is associated with convection $\left(\left\langle P_{\text {con }}^{(\nu)}\right\rangle\right)$, as given by the relation ${ }^{10}$

$$
P_{\text {con }}^{(\nu)}=2.09 \delta S_{\nu} \sum_{i}\left(T_{i}-T_{\nu}\right)\left|T_{i}-T_{\nu}\right|^{0.25}
$$

where $\delta$ is a dimensionless parameter that has been introduced to account for the relative importance of convection in the overall energy balance. Substituting Eqs. (3)-(8) into Eq. (2), we obtain the detailed heat-dynamics equation for layer $v$ :

$$
C_{\rho_{\nu}} \rho_{\nu} V_{\nu}\left(\frac{\mathrm{d} T_{\nu}}{\mathrm{d} t}\right)=\left\langle P_{\mathrm{mw}}\right\rangle_{\nu}+\left\langle P_{\mathrm{rad}_{\nu}}\right\rangle+P_{\mathrm{con}_{\nu}}
$$

As already indicated, we consider only the slow time variations, which correspond to changes in the temperature of the material $(T(t))$ and the convection power, so that the microwave radiation is averaged. Equation (9) is a nonlinear differential equation for the temperature of each layer. Solving this equation for each layer gives the temperature of each layer as a function of time, which is the main goal of this study.

\section{Simulation Results}

Simulations of Eq. (9) were performed for $T_{\mathrm{Al}_{2} \mathrm{O}_{3}}(t=0)=$ $T_{\mathrm{ZrO}_{2}}(t=0)=T_{\mathrm{SiC}}(t=0)=25^{\circ} \mathrm{C}, f=2.45 \mathrm{GHz}$, and the following material mass densities: $\rho_{\mathrm{ZrO}_{2}}=2520 \mathrm{~kg} / \mathrm{m}^{3}, \rho_{\mathrm{Al}_{2} \mathrm{O}_{3}}=$ $1588 \mathrm{~kg} / \mathrm{m}^{3}$, and $\rho_{\mathrm{SiC}}=965 \mathrm{~kg} / \mathrm{m}^{3}$. A $\delta^{2}$ value of 0.9 was selected, based on previous work, ${ }^{7}$ and typical values were used for the emissivity: $\hat{\varepsilon}_{\mathrm{Al}_{2} \mathrm{O}_{3}}=0.01, \hat{\varepsilon}_{\mathrm{SiC}}=0.1$, and $\hat{\varepsilon}_{\mathrm{ZrO}_{2}}=0.99$ (all values were assumed to be independent of temperature). The dielectric widths of the materials were assumed to be $d_{\mathrm{ZrO}_{2}}=7.5 \mathrm{~mm}, d_{\mathrm{Al}_{2} \mathrm{O}_{3}}$ $=12.5 \mathrm{~mm}$, and $d_{\mathrm{SiC}}=10 \mathrm{~mm}$. As indicated in Fig. 1, the system is composed of seven consecutive dielectric layers $\left(\mathrm{Al}_{2} \mathrm{O}_{3}, \mathrm{SiC}\right.$, $\mathrm{Al}_{2} \mathrm{O}_{3}, \mathrm{ZrO}_{2}, \mathrm{Al}_{2} \mathrm{O}_{3}, \mathrm{SiC}$, and $\mathrm{Al}_{2} \mathrm{O}_{3}$ ). For this arrangement, the factor matrix $[\xi]_{i, v=1 \ldots 7}$ that was introduced in Eq. (7) is given by

$$
\xi=\left(\begin{array}{ccccccc}
1 & 6 / 21 & 5 / 21 & 4 / 21 & 3 / 21 & 2 / 21 & 1 / 21 \\
6 / 26 & 1 & 6 / 26 & 5 / 26 & 4 / 26 & 3 / 26 & 2 / 26 \\
5 / 29 & 6 / 29 & 1 & 6 / 29 & 5 / 29 & 4 / 29 & 3 / 29 \\
4 / 30 & 5 / 30 & 6 / 30 & 1 & 6 / 30 & 5 / 30 & 4 / 30 \\
3 / 29 & 4 / 29 & 5 / 29 & 6 / 29 & 1 & 6 / 29 & 5 / 29 \\
2 / 26 & 3 / 26 & 4 / 26 & 5 / 26 & 6 / 26 & 1 & 6 / 26 \\
1 / 21 & 2 / 21 & 3 / 21 & 4 / 21 & 5 / 21 & 6 / 21 & 1
\end{array}\right)
$$

The values of $\xi$ were determined by the actual power incident from material $i$ to layer $v$ ( see Skamser et $a l .^{8}$ ); however, the model is not very sensitive to these values. We used the above-described conditions for each material and, using time steps of $0.1 \mathrm{~s}$, we calculated the corresponding power source (using the corresponding dielectric coefficient, as in Fig. 2); then, using Eq. (9), we updated the corresponding temperature $T(t)$ for each time step. However, the strongly nonlinear character of the problem may require quite different time steps for other configurations.

Figure 3 shows the time variation of the temperature of $\mathrm{ZrO}_{2}$, $\mathrm{SiC}$, and $\mathrm{Al}_{2} \mathrm{O}_{3}$. The curves for $\mathrm{ZrO}_{2}$ and $\mathrm{Al}_{2} \mathrm{O}_{3}$ show no rapid initial temperature increase; however, after some period of time, the $\mathrm{ZrO}_{2}$ shows a steep increase in temperature that exceeds that of the $\mathrm{SiC}$ at high temperatures. As expected, $\mathrm{Al}_{2} \mathrm{O}_{3}$ shows only a small increase in temperature, because it has poor microwave absorption. Figure 4 shows how the time of intersection $\left(\tau_{c}\right)$, for $\mathrm{ZrO}_{2}$ and $\mathrm{SiC}$ at equal temperature, increases as a function of the $\mathrm{ZrO}_{2}$ volume (which corresponds to the thickness in our model) and decreases as a function of its emissivity $\hat{\varepsilon}$. An empirical best fit suggests that the intersection time (given in minutes) is dependent on the volume (given in cubic centimeters) as

$$
\tau_{c}(V)=86 V^{2}+30 V+4
$$

whereas its dependence on the emissivity varies as

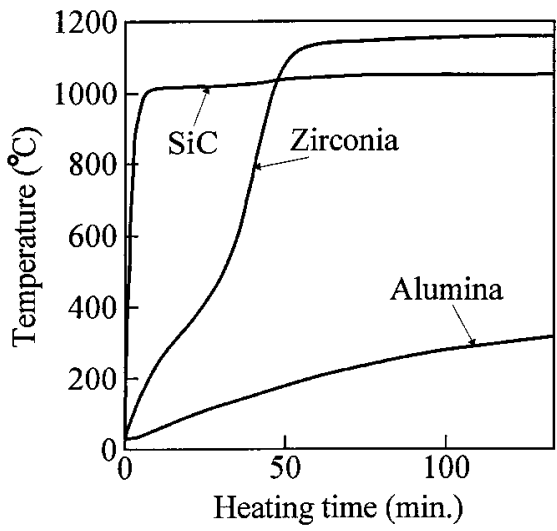

Fig. 3. Temperature-time curves for $\mathrm{ZrO}_{2}, \mathrm{SiC}$, and $\mathrm{Al}_{2} \mathrm{O}_{3}$ under the following conditions: $f=2.45 \mathrm{GHz}, \rho_{\mathrm{ZrO}_{2}}=2520 \mathrm{~kg} / \mathrm{m}^{3}, \rho_{\mathrm{Al}_{2} \mathrm{O}_{3}}=1588$ $\mathrm{kg} / \mathrm{m}^{3}, \rho_{\mathrm{SiC}}=965 \mathrm{~kg} / \mathrm{m}^{3}, d_{\mathrm{ZrO}_{2}}=7.5 \mathrm{~mm}, d_{\mathrm{Al}_{2} \mathrm{O}_{3}}=12.5 \mathrm{~mm}$, and $d_{\mathrm{SiC}}=$ $10 \mathrm{~mm}$.


Fig. 4. Dependence of the intersection time between $\mathrm{ZrO}_{2}$ and $\mathrm{SiC}$ on (a) the $\mathrm{ZrO}_{2}$ volume and (b) the $\mathrm{ZrO}_{2}$ emissivity.

$$
\tau_{\mathrm{c}}(\hat{\varepsilon})=37 \exp (-4 \hat{\varepsilon})+47
$$

This last result could provide a simple method to determine the material emissivity from an experiment that is based on the present model. In principle, the applied power also affects the intersection time $\left(\tau_{c}\right)$ for a specific configuration; here, we tacitly have assumed that this power is set by the characteristics of the furnace.

One advantage of the present model is that each mechanism (blackbody radiation and convection) can be turned on and off, to examine the effect of each process on the energy exchange. Figure 5 demonstrates the role of blackbody radiation in the energytransfer process. Figure 5(a) shows the temperature dynamics when all the processes that contribute to the energy flow are considered. Without blackbody radiation, the temperature of the $\mathrm{SiC}$ increases rapidly to high values, whereas the $\mathrm{ZrO}_{2}$ temperature increases only slightly (Fig. 5(b)). Figure 5(c) illustrates the temperature variation with time when the blackbody effect is "turned off" after $30 \mathrm{~min}$; the temperature of the SiC then increases rapidly, because the primary "cooling" mechanism has been removed, and no significant effect is observed in the $\mathrm{ZrO}_{2}$.

Figure 6 further demonstrates the influence of blackbody radiation and shows the contribution of each factor ( $r$ field, 


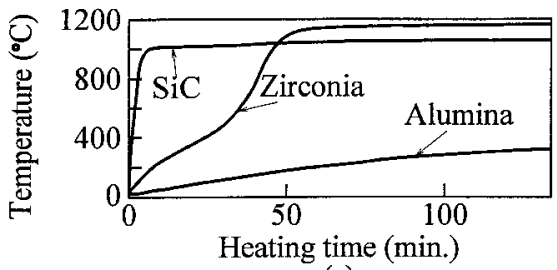

(a)

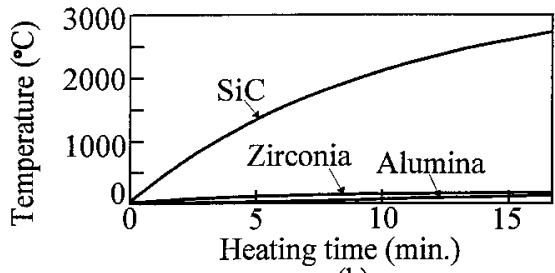

(b)

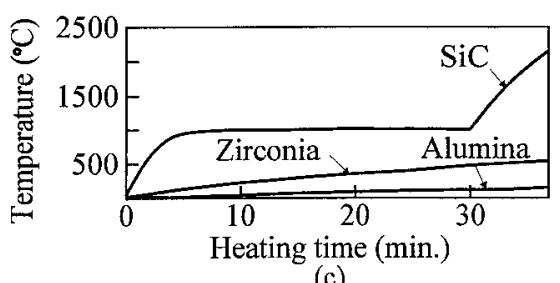

(c)

Fig. 5. Temperature-time curves (a) with the impact of the blackbody radiation, (b) without the impact of the blackbody radiation, and (c) neutralizing the impact of the blackbody radiation after $30 \mathrm{~min}$ (before the intersection point).

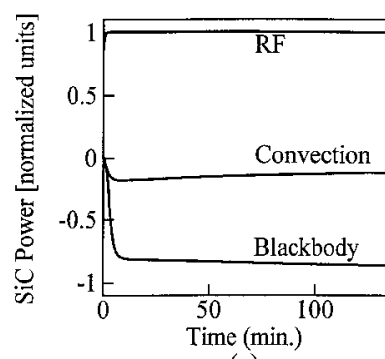

(a)

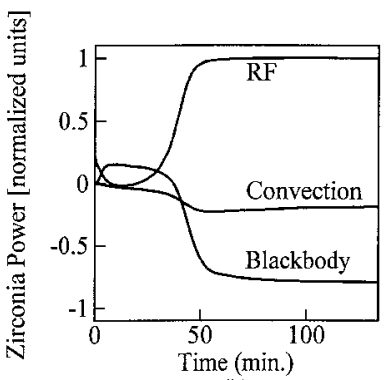

(b)
Fig. 6. Contribution of the rf field, convection, and blackbody powers, each as a function of time, for (a) $\mathrm{SiC}$ and (b) $\mathrm{ZrO}_{2}$. Note the shift in the role of blackbody radiation in the case of $\mathrm{ZrO}_{2}$ : in the first period of time, power is absorbed, whereas in the second period of time, power is dissipated.

convection, and blackbody) on the temperatures of the $\mathrm{ZrO}_{2}$ and $\mathrm{SiC}$ under the same conditions as those in Fig. 3. The top frame shows that the rf field continuously contributes to the heating of the $\mathrm{SiC}$, whereas the blackbody radiation is responsible for its "cooling." Convection has a relatively minor role. The bottom frame illustrates the temperature dynamics in $\mathrm{ZrO}_{2}$. During the initial period, the rf-field contribution to the temperature increase is negligible, and the main contribution originates from blackbody radiation that is emitted by the $\mathrm{SiC}$. Subsequently, the rf field dominates the temperature increase, whereas the blackbody radiation becomes a cooling mechanism.

The model was tested against previously reported experimental results. ${ }^{7}$ Figure 7 compares the experimental and simulated temperature-time curves for various microwave-input powers. Reasonable agreement of the simulation with the experimental data is observed, especially for SiC. Discrepancies are probably either due to the fact that our study used a 1-D model, whereas the experiment used a 3-D model, or due to inaccuracies that are associated with the parametric description of the dielectric coefficients, as a function of temperature.

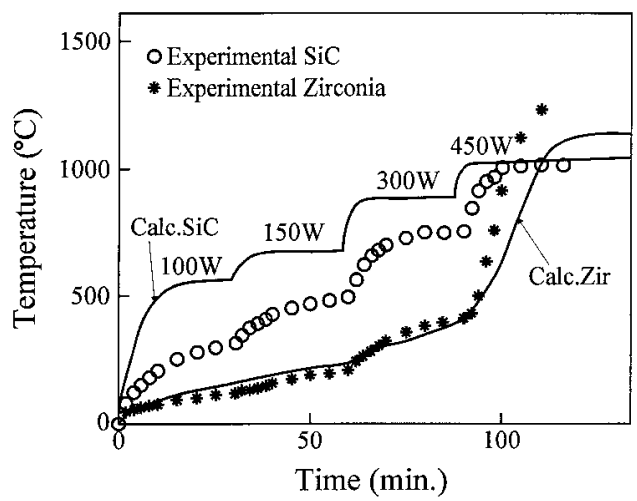

Fig. 7. Experimental and calculated temperature-time curves for $\mathrm{ZrO}_{2}$ and $\mathrm{SiC}$.

\section{Conclusions}

A quasi-analytical model has been developed to examine the energy conversion during the microwave sintering of a ceramic that is surrounded by a secondary heater. The present theoretical model accounts for multiple reflections in all sections of the system, to evaluate the energy flow between the various regions. These aspects are critical for the evaluation of the power that is deposited by the microwave field in each layer when the temperature-and, thus, the dielectric coefficient-vary rapidly. The effect of each energy-exchange process on the temperature dynamics has been examined. Blackbody radiation has the dominant role, whereas convection has a secondary role. Simulation results and previously reported experimental data agree well. The model allows determination of the time required to equalize the temperature of the sintered sample and the susceptor, thus providing, in this case, significant design information for the optimal sintering system. The model that has been described here can be further extended using a three-dimensional model and giving consideration to the spatial variation of the temperature in each of the material layers.

\section{Acknowledgment}

During the preparation of this study, the authors benefited from the useful comments of Prof. D. Brandon.

\section{References}

${ }^{1}$ W. H. Sutton, "Microwave Processing of Ceramic Materials," Am. Ceram. Soc. Bull., 68 [2] 376-86 (1989).

${ }^{2}$ Y.-L. Tian, B.-S. Li, J.-L. Shi, Y.-P. Xu, J.-K. Guo, and D.-S. Yen, "Microwave Sintering of $\mathrm{Y}_{2} \mathrm{O}_{3}(3 \%)-\mathrm{ZrO}_{2}$ (TZP)"; pp. 577-84 in Ceramic Transactions, Vol. 21, Microwaves: Theory and Application in Materials Processing. Edited by D. E. Clark, F. D. Gac, and W. H. Sutton. American Ceramic Society, Westerville, OH, 1991.

${ }^{3}$ J. D. Katz, R. D. Blake, and J. J. Petrovic, "Microwave Sintering of AluminaSilicon Carbide Composites at 2.45 and 60 GHz," Ceram. Eng. Sci. Proc., 9 [7-8] 725-34 (1988).

${ }^{4}$ P. D. Ramesh, Ph.D Thesis. Indian Institute of Science, Bangalore, India, 1995.

${ }^{5}$ A. Dé, I. Ahmad, E. D. Whitney, and D. E. Clark, "Microwave (Hybrid) Heating of Alumina at $2.45 \mathrm{GHz}$ : I. Microstructural Uniformity and Homogeneity"; see Ref. 2, pp. $319-28$.

${ }^{6}$ M. A. Janney, C. L. Calhoun, and H. D. Kimrey, "Microwave Sintering of Solid Oxide Fuel Cell Materials: Zirconia-8\% mol\% Yttria," J. Am. Ceram. Soc., 75 [2] 341-46 (1992).

${ }^{7}$ P. D. Ramesh, D. Brandon, and L. Schächter, "Use of Partially Oxidized SiC Particle Bed for Microwave Sintering of Low Loss Ceramics," Mater. Sci. Eng. A, A266 [1-2] 211-20 (1999).

${ }^{8}$ D. J. Skamser, J. J. Thomas, H. M. Jennings, and D. L. Johnson, "A Model for Microwave Processing of Compositionally Changing Ceramic System," J. Mater. Res., 10, 3160-78 (1995).

${ }^{9}$ D. T. Haar and H. Wergeland, Elements of Thermodynamics; pp. 369-477 (Ch. 19). Addison-Wesley Press, Reading, MA, 1966.

${ }^{10}$ H. S. Carslaw and J. C. Jaeger, Conduction of Heat in Solids; 2nd Ed. Oxford University Press, NJ, 1959. 